\title{
Clinical, biochemical, and genetic analysis of a Korean neonate with hereditary tyrosinemia type 1
}

\author{
Hyung-Doo Park', Dong Hwan Lee², Tae-Youn \\ Choi $^{3}$, You Kyoung Lee ${ }^{4}$, Jong-Won Kim', \\ Chang-Seok $\mathrm{Ki}^{1, *}$ and Yong-Wha Lee ${ }^{4, *}$ \\ ${ }^{1}$ Department of Laboratory Medicine and Genetics, \\ Samsung Medical Center, Sungkyunkwan University \\ School of Medicine, Seoul, Korea \\ ${ }^{2}$ Department of Pediatrics, Soonchunhyang \\ University College of Medicine, Seoul, Korea \\ ${ }^{3}$ Department of Laboratory Medicine, \\ Soonchunhyang University Hospital and \\ Soonchunhyang University College of Medicine, \\ Seoul, Korea \\ ${ }^{4}$ Department of Laboratory Medicine and Genetics, \\ Soonchunhyang University Bucheon Hospital and \\ Soonchunhyang University College of Medicine, \\ Bucheon, Korea
}

\begin{abstract}
Background: Hereditary tyrosinemia type 1 (HT1; MIM 276700 ) is caused by mutations in the fumarylacetoacetate hydrolase $(F A H)$ gene, and is the most severe disorder associated with the tyrosine catabolic pathway. HT1 is a very rare disorder and no genetically confirmed case of HT1 in Korea has yet been reported. In this study, we present a Korean neonate with clinical and biochemical features of HT1.

Methods: A female neonate was admitted to our hospital for further work-up of an abnormal newborn screening test. We analyzed amino acids and organic acids in the patient's blood and urine. To confirm the presence of the genetic abnormality, all the coding exons of the $F A H$ gene and the flanking introns were amplified by polymerase chain reaction (PCR).

Results: The patient's newborn screening test revealed increased concentrations of methionine and tyrosine. Subsequent urine organic acid analysis showed increased urinary excretion of 4-hydroxyphenyllactate, 4-hydroxyphenylpyruvate, succinate, and succinylacetone. Gap-PCR and sequence analysis of the $F A H$ gene revealed a homozygous large deletion mutation encompassing exons 12-14. The patient's
\end{abstract}

*Corresponding authors: Yong-Wha Lee, MD, PhD, Department of Laboratory Medicine and Genetics, Soonchunhyang University Bucheon Hospital and Soonchunhyang University College of Medicine, 1174 Jung-dong, Wonmi-gu, Bucheon, 420-767, Korea Phone: +82-32-621-5943, Fax: +82-32-621-5944, E-mail: lywmd@schbc.ac.kr

Chang-Seok Ki, MD, PhD, Department of Laboratory Medicine and Genetics, Samsung Medical Center, Sungkyunkwan University School of Medicine, 50 Irwon-

Dong, Gangnam-Gu, Seoul, 135-710, Korea

Phone: +82-2-3410-2709, Fax: +82-2-3410-2710,

E-mail: changski@skku.edu

Received March 8, 2009; accepted May 18, 2009;

previously published online July 2, 2009 parents were not consanguineous but were heterozygous carriers of the same mutation.

Conclusions: The patient had a novel, large deletion mutation of $F A H$ and is the first report of genetically confirmed HT1 in Korea.

Clin Chem Lab Med 2009;47:930-3.

Keywords: fumarylacetoacetate hydrolase $(F A H)$; Korean; large deletion; novel mutation; tyrosinemia.

\section{Introduction}

Hereditary tyrosinemia type I (HT1, MIM 276700), also referred to as hepato-renal tyrosinemia, is the most severe disorder linked to the tyrosine catabolic pathway (1). HT1 is caused by decreased activity of fumarylacetoacetate hydrolase (FAH; EC 3.7.1.2), which is the last enzyme that hydrolyzes fumarylacetoacetate to fumarate and acetoacetate in the tyrosine catabolic pathway $(2,3)$. FAH deficiency causes severe progressive liver disease in infancy, renal tubular defects with hypophosphatemic rickets, and neurologic crises (2). If not treated, patients usually die of liver failure within the first year of life.

The worldwide prevalence of HT1 is known to be very low (1:100,000-1:120,000 births); its prevalence in Koreans is not known $(4,5)$. The human $F A H$ gene maps to the long arm of chromosome 15 in the region q23-q25 and contains 14 exons that encode $F A H$ mRNA with a length of at least 1477 nucleotides (6, 7). There are variable levels of $\mathrm{FAH}$ enzyme activity in liver tissue from patients with HT1 (8). The relationship between genotype and phenotype is unclear, because different clinical presentations of HT1 have been reported in patients with identical genotypes (9). Genotype heterogeneity is not sufficient for explaining the clinical heterogeneity, and other factors may modify the phenotype in HT1 (9).

In this report, we present the first biochemically and genetically confirmed Korean patient with HT1. Interestingly, the patient was homozygous for a novel large deletion mutation of the $F A H$ gene.

\section{Materials and methods}

\section{Clinical and biochemical analysis}

\footnotetext{
A female neonate was admitted to our hospital for further evaluation of an abnormal newborn screening test performed 2 days after birth at a local hospital. At 1 month of age, the patient underwent various biochemical tests, including total protein, aspartate aminotransferase, alanine aminotransferase, alkaline phosphatase, ammonia, albumin, bilirubin and lactate. In addition, tests in the newborn screening test were confirmed by liquid chromatography-
} 
tandem mass spectrometry (LC-MS/MS), and amino acids and organic acids in the blood and urine were analyzed. Abdominal ultrasonography was performed to visualize abdominal anatomical structures.

\section{Genetic analysis}

The study was approved by the Ethical Committee of our institution. Blood samples were collected from the patient and parents after obtaining informed parental consent. Genomic DNA was isolated from peripheral blood leukocytes using the Wizard Genomic DNA Purification Kit (Promega, Madison, WI, USA). All of the coding exons of the $F A H$ gene and flanking introns were amplified using polymerase chain reaction (PCR) with primers designed by the authors (sequences available upon request) and a thermal cycler (Model 9700; Applied Biosystems, Foster City, CA, USA). Five microliters of amplification product were treated with $10 \mathrm{U}$ shrimp alkaline phosphatase and $2 \mathrm{U}$ exonuclease I (USB Corp., Cleveland, OH, USA). Direct sequencing was performed with the ABI Prism 3100 Genetic Analyzer (Applied Biosystems) using the BigDye Terminator Cycle SequencingReady Reaction Kit (Applied Biosystems). All novel mutations were confirmed by testing 100 control chromosomes.

\section{Gap-PCR}

Gap-PCR using the AccuPower TLA PCR premix (Bioneer, Daejeon, Korea) was performed since the patient had a large deletion in the $F A H$ gene. Using chromosomal walking with different pairs of primers, the smallest PCR amplicon (expected size: 18,453 bp) was detected using the primer pair: forward $\left(5^{\prime}\right.$-accctgttgctctttgcagt- $\left.3^{\prime}\right)$ and reverse $\left(3^{\prime}\right.$-gaaggagtcacccaatggaa- $\left.5^{\prime}\right)$. The primer sequences were based on the intron sequences of the $F A H$ gene obtained from the Ensembl Genome Browser (http://www.ensembl. org).

\section{Results}

\section{Clinical and biochemical findings}

The patient was born at 40 weeks' gestation by vaginal delivery and weighed $3.48 \mathrm{~kg}$. Physical examination revealed an asymmetric face and hyperflexible wrist joints. A repeat newborn screening test at 9 days of life showed increased concentrations of phenylalanine, methionine and tyrosine: $155 \mu \mathrm{M}$ phenylalanine (cut-off, $\leq 125 \mu \mathrm{M}$ ), $199 \mu \mathrm{M}$ methionine (cut-off, $\leq 67 \mu \mathrm{M}$ ) and $481 \mu \mathrm{M}$ tyrosine (cut-off, $\leq 305 \mu \mathrm{M}$ ).

Liver function tests performed at 1 month of age were also abnormal. Aspartate aminotransferase was 0.94 [reference range $(R R)<0.53$ ] $\mu \mathrm{kat} / \mathrm{L}$, alkaline phosphatase was 1650 (RR, 42-98) U/L, ammonia was $89.3(\mathrm{RR}, 17.9-46.4) \mu \mathrm{mol} / \mathrm{L}$, and lactate was 6.5 (RR, 0.5-2.2) $\mathrm{mmol} / \mathrm{L}$. Urine organic acid analysis at 1 month of age showed increased urinary excretion of 4-hydroxyphenyllactate, 4-hydroxyphenylpyruvate, succinate, and succinylacetone [4-hydroxyphenyllactate: $893 \mathrm{mmol} / \mathrm{mol}$ creatinine (cut-off, <3), 4hydroxyphenylpyruvate: $563 \mathrm{mmol} / \mathrm{mol}$ creatinine (cut-off, $<1$ ), succinate: $370 \mathrm{mmol} / \mathrm{mol}$ creatinine (cutoff, $<79)$, and succinylacetone: $28 \mathrm{mmol} / \mathrm{mol}$ creatinine (cut-off, non-detected)]. Abdominal ultrasound revealed ascites and coarse parenchymal changes in the liver.

The patient was diagnosed with HT1 based on clinical and biochemical tests at 47 days after birth. Treatment with 2-(2-nitro-4-trifluoromethylbenzoyl)1,3-cyclohexanedione (NTBC) and dietary tyrosine restriction was initiated after confirmation of tyrosinemia type I. Following 1 week of treatment, the patient's increased urinary succinylacetone declined to non-detectable concentrations and remained $<1 \mathrm{mmol} / \mathrm{mol}$ creatinine during follow-up. Urinary concentrations of methionine and tyrosine reverted to normal values of $5 \mu \mathrm{M}$ and $11 \mu \mathrm{M}$, respectively, by 2 months of age. The patient's disease was well controlled, and she was seen as an outpatient.

At 4 months after birth, the patient developed acute symptoms, including uncontrolled fever and dyspnea suggestive of sepsis secondary to aspiration pneumonia. This developed independently of the patient's tyrosinemia. Although prompt management and close observation was performed, septic complications including metabolic acidosis, ascites, and disseminated intravascular coagulation developed and the patient expired following cardiac arrest.

\section{Genetic analysis}

PCR of each $F A H$ exon with intron primer failed to amplify exons 12,13, and 14, suggesting that the patient was homozygous for a large deletion. Using different primer pairs that covered the region from intron 11 of the FAH gene to the FAH-ARNT2 intergenic region, gap-PCR was performed to identify the breakpoint of the large deletion of exons 12, 13 and 14 (Figure 1). Sequence analysis of the PCR amplicon revealed an 18,036 bp deleted region that spanned from nucleotide 1130 of intron 11 of the $F A H$ gene to nucleotide 10,539 of the FAH-ARNT2 intergenic region. According to the numbering position of the human $F A H$ reference sequence, the mutation could

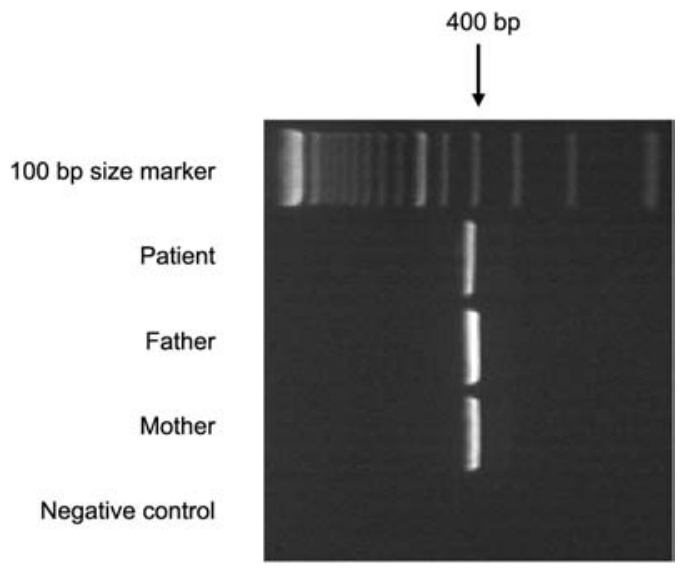

Figure 1 Gap-PCR results of the $F A H$ gene.

Gap-PCR encompassing exons 12,13 and 14 of the $F A H$ gene resulted in an expected amplicon of $18.5 \mathrm{~kb}$, which was not amplified from a control sample. A shorter 417 bp product was detected in the patient, who has a homozygous large deletion. The patient's parents were carriers of this large deletion. 
be described as c. $960+1130$ * $1260+10539$ del 18036 (reference sequence from NC_000015.8 and NM_ 000137.1) (Figure 2). The patient's parents were carriers of this large deletion, and the patient inherited one mutant allele from each parent. Screening of 50 normal controls revealed no mutant allele in 100 chromosomes.

\section{Discussion}

Currently, most laboratories perform newborn screening tests using dried blood spots analyzed with LC-MS/MS. However, this is a limited screening method for HT1 because tyrosine is increased in cases of benign transient tyrosinemia. In addition, the increased concentration of tyrosine in patients with HT1 may overlap the normal range in control populations (4). Therefore, we determined the concentration of succinylacetone, a specific marker for HT1, in dried blood spots using LC-MS/MS (10). The concentrations of methionine and tyrosine in this case were not dramatically increased, but we diagnosed HT1 with the results from the analysis of urine organic acids.

Although there have been a few reports of Korean patients with tyrosinemia type 1 (11-13), all cases were diagnosed by clinical and biochemical findings without investigation of the molecular characteristics. This is the first case in Korea of HT1 that was con- firmed with genetic analysis in addition to the biochemical abnormalities. In addition, the large deletion identified in this study is a novel mutation. The most common FAH gene mutations in patients with HT1 differ with respect to ethnicity. For example, the specific mutation IVS12 $+5 \mathrm{G}>\mathrm{A}$ is prevalent in French Canadian and Scandinavian, excluding Finnish, populations $(14,15)$. In addition, five mutations IVS6$1 \mathrm{G}>\mathrm{T}$, c.1009G $>\mathrm{A}, \quad$ c.192G $>\mathrm{T}$ (a splicing error), p.D233V, and p.W262X are common in Central and Western Europe, Scandinavia, Pakistan, Turkey, and Finland, respectively (15-18). These mutations are thought to have spread by the founder effect.

In the $F A H$ gene, missense and nonsense point mutations, and splicing errors are common and account for $90 \%$ of the total number of mutations reported in the Human Gene Mutation Database (HGMD) at the Institute of Medical Genetics in Cardiff (http://www.hgmd.cf.ac.uk/ac/gene.php?gene=FAH). Large deletions of the $F A H$ gene have rarely been reported. Only one mutant allele with a large deletion, E6/16del26 (c.548_553+20del), has been reported previously (17). In the present study, we identified a novel mutation, c. $960+1130$ * $1260+10539$ del18036, resulting in the loss of 100 residues (amino acids 321-420) from the protein. It was also interesting that the patient's parents were heterozygous carriers of the same allele with a large deletion of the $F A H$ gene. The presence of a homozygous deletion in a non-consanguineous pedigree is very unusual.

Chromosome 15q25
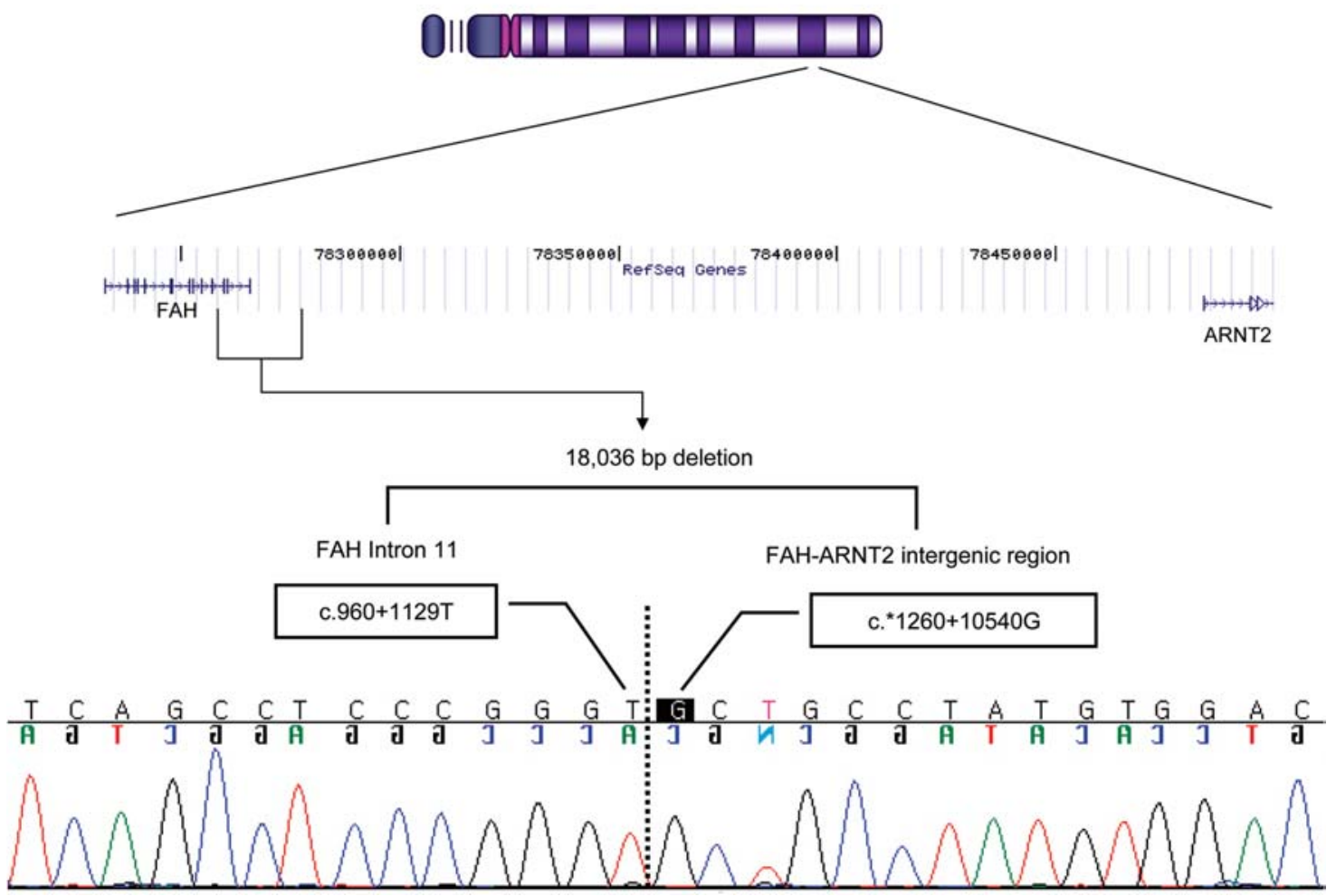

Figure 2 Breakpoint analysis of the deletion of exons 12-14 of the FAH gene.

Sequence analysis shows a deletion of 18,036 bp that spans from nucleotide 1130 of intron 11 of the $F A H$ gene to nucleotide 10,539 of the FAH-ARNT2 intergenic region, designated as c.960+1130_*1260+10539del18036 (reference sequence from NC_000015.8 and NM_000137.1). 
Although the patient temporarily improved after administration of NTBC, she died at 4 months of age due to aspiration pneumonia and sepsis. NTBC is a potent inhibitor of 4-hydroxyphenylpyruvate dioxygenase and has improved the outcomes of most patients with hereditary tyrosinemia (19). The patient was diagnosed clinically with an acute form of HT1, given her presentation of hepatic signs and symptoms within the first 6 months of life (20). This clinical presentation may be due to the large $F A H$ deletion, containing exons $12-14$, which lead to the synthesis of structurally unstable, incomplete proteins, thereby inducing attenuation of FAH activity.

In summary, we diagnosed a Korean patient with HT1 using biochemical and molecular analysis, revealing a novel large-deletion $F A H$ mutation. The gap-PCR method developed in this study may be a useful tool for the identification of Korean HT1 patients with deletions of exons 12-14.

\section{References}

1. St-Louis M, Tanguay RM. Mutations in the fumarylacetoacetate hydrolase gene causing hereditary tyrosinemia type I: overview. Hum Mutat 1997;9:291-9.

2. Kvittingen EA. Hereditary tyrosinemia type I - an overview. Scand J Clin Lab Invest Suppl 1986;184:27-34.

3. Lindblad B, Lindstedt S, Steen G. On the enzymic defects in hereditary tyrosinemia. Proc Natl Acad Sci USA 1977; 74:4641-5.

4. Mitchell GA, Grompe M, Lambert M, Tanguay RM. Hypertyrosinemia. In: Scriver CR, Beaudet AL, Sly WS, Valle D, editors. The metabolic and molecular bases of inherited disease. NY: McGraw Hill, 2001:1777-806.

5. Bergeron A, D'Astous M, Timm DE, Tanguay RM. Structural and functional analysis of missense mutations in fumarylacetoacetate hydrolase, the gene deficient in hereditary tyrosinemia type 1. J Biol Chem 2001;276: 15225-31.

6. Phaneuf D, Labelle Y, Berube D, Arden K, Cavenee W, Gagne $R$, et al. Cloning and expression of the cDNA encoding human fumarylacetoacetate hydrolase, the enzyme deficient in hereditary tyrosinemia: assignment of the gene to chromosome 15. Am J Hum Genet 1991; 48:525-35.

7. Ploos van Amstel JK, Bergman AJ, van Beurden EA, Roijers JF, Peelen $T$, van den Berg IE, et al. Hereditary tyrosinemia type 1: novel missense, nonsense and splice consensus mutations in the human fumarylacetoacetate hydrolase gene; variability of the genotype-phenotype relationship. Hum Genet 1996;97:51-9.

8. Kvittingen EA, Rootwelt $H$, Brandtzaeg $P$, Bergan $A$, Berger R. Hereditary tyrosinemia type I. Self-induced correction of the fumarylacetoacetase defect. J Clin Invest 1993;91:1816-21.

9. Poudrier J, Lettre F, Scriver CR, Larochelle J, Tanguay RM. Different clinical forms of hereditary tyrosinemia (type I) in patients with identical genotypes. Mol Genet Metab 1998;64:119-25.

10. Magera MJ, Gunawardena ND, Hahn SH, Tortorelli S, Mitchell GA, Goodman SI, et al. Quantitative determination of succinylacetone in dried blood spots for newborn screening of tyrosinemia type I. Mol Genet Metab 2006;88:16-21.

11. Cho JH, Shim KJ, Kim SK, Shin SH, Lee KH, Yun HS. A case of tyrosinemia type 1 with cytomegalovirus infection. Korean J Pediatr 2004;47:111-4.

12. Hahn SH, Pai KS, Lee KB, Park KH, Kim OH, Hong $\mathrm{CH}$, et al. Acute tyrosinemia type 1 in a 5-month-old Korean boy. J Korean Pediatr Soc 1996;39:866-72.

13. Kim KT, Kim YM, Park SE, Nam SO, Park JH. Two cases of acute form of tyrosinemia type I. J Korean Pediatr Soc 2002;45:131-6.

14. Grompe $M$, St-Louis $M$, Demers $\mathrm{SI}$, al-Dhalimy $\mathrm{M}$, Leclerc B, Tanguay RM. A single mutation of the fumarylacetoacetate hydrolase gene in French Canadians with hereditary tyrosinemia type I. N Engl J Med 1994; 331:353-7.

15. Rootwelt $H$, Hoie K, Berger R, Kvittingen EA. Fumarylacetoacetase mutations in tyrosinaemia type I. Hum Mutat 1996;7:239-43.

16. Scott CR. The genetic tyrosinemias. Am J Med Genet C Semin Med Genet 2006;142C:121-6.

17. Arranz JA, Pinol F, Kozak L, Perez-Cerda C, Cormand B, Ugarte $M$, et al. Splicing mutations, mainly IVS6-1 (G $>$ T), account for $70 \%$ of fumarylacetoacetate hydrolase (FAH) gene alterations, including 7 novel mutations, in a survey of 29 tyrosinemia type I patients. Hum Mutat 2002;20:180-8.

18. Rootwelt H, Berger R, Gray G, Kelly DA, Coskun T, Kvittingen EA. Novel splice, missense, and nonsense mutations in the fumarylacetoacetase gene causing tyrosinemia type 1. Am J Hum Genet 1994;55:653-8.

19. Lindstedt S, Holme E, Lock EA, Hjalmarson O, Strandvik B. Treatment of hereditary tyrosinaemia type I by inhibition of 4-hydroxyphenylpyruvate dioxygenase. Lancet 1992;340:813-7.

20. Bergman AJ, van den Berg IE, Brink W, Poll-The BT, Ploos van Amstel JK, Berger R. Spectrum of mutations in the fumarylacetoacetate hydrolase gene of tyrosinemia type 1 patients in northwestern Europe and Mediterranean countries. Hum Mutat 1998;12:19-26. 